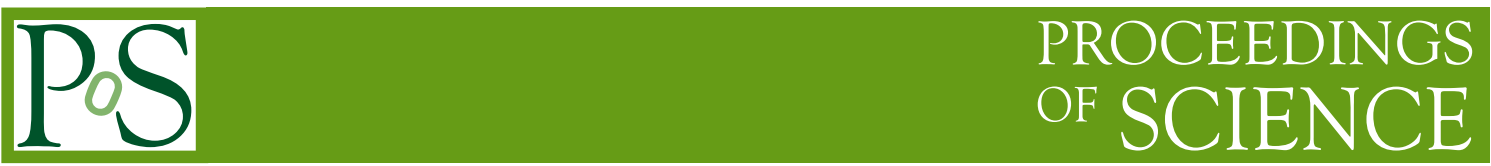

\title{
Muon (g-2) anomaly in extended left-right symmetric model
}

Prativa Pritimita, ${ }^{a, *}$ Chayan Majumdar, ${ }^{a}$ Sudhanwa Patra, ${ }^{b}$ Supriya Senapati ${ }^{a}$ and Urjit A. Yajnik $^{a}$

${ }^{a}$ Indian Institute of Technology Bombay,

Powai, Mumbai, India 400076

${ }^{b}$ Indian Institute of Technology Bhilai,

Sejbahar, Chattisgarh, India 492015

E-mail: prativa@iitb.ac.in

We consider a gauged $U(1)_{L_{\mu}-L_{\tau}}$ extension of the left-right symmetric theory in order to simultaneously explain neutrino mass, mixing and muon anomalous magnetic moment. We get sizeable contribution from the interaction of the new light gauge boson $Z_{\mu \tau}$ of $U(1)_{L_{\mu}-L_{\tau}}$ symmetry with muons which can individually satisfy the current bounds on muon $(g-2)$ anomaly . The other positive contributions to $\Delta a_{\mu}$ come from the interactions of neutral CP-even scalars with muons and that of singly charged gauge boson $W_{L}$ with heavy neutrinos which is facilitated by inverse seesaw mechanism that explains neutrino mass in our model. The results show that the model gives a small but non-negligible contribution to $\Delta a_{\mu}$ thereby eliminating the entire deviation in theoretical prediction and experimental result of muon $(g-2)$ anomaly.

40th International Conference on High Energy physics - ICHEP2020

July 28 - August 6, 2020

Prague, Czech Republic (virtual meeting)

\footnotetext{
${ }^{*}$ Speaker
} 
The Standard Model (SM) predicts the muon anomalous magnetic moment to be $a_{\mu}^{\mathrm{SM}}=$ $(11659183.0 \pm 4.8) \times 10^{-10}[1,2]$, whereas its precise measurement by Brookhaven National Laboratory (BNL) is $a_{\mu}^{\text {exp }}=(11659209.1 \pm 6.3) \times 10^{-10}[1,3]$ with $\Delta a_{\mu}=(26.1 \pm 7.9) \times 10^{-10}$ [4]. Many new physics scenarios have been explored in attempt to eliminate or narrow down this wide gap in prediction and measurement [5-15] some of which focus on $U(1)_{L_{\mu}-L_{\tau}}$ symmetry. The gauged $U(1)_{L_{\mu}-L_{\tau}}$ symmetry is popular since the parameters associated with its gauge boson $Z_{\mu \tau}$ are not constrained by lepton and hadron colliders as it doesn't couple to electrons and quarks. Moreover, as per the constraints given by neutrino-trident experiments [16] a low mass of $O(100$ $\mathrm{MeV}$ ) can be allowed for $Z_{\mu \tau}$ for a coupling as low as $g_{\mu \tau} \leq 10^{-3}$. With the motivation of explaining neutrino mass, mixing and muon $(g-2)$ anomaly in a single framework we have augmented the Leftright symmetric model (LRSM) with $U(1)_{L_{\mu}-L_{\tau}}$ symmetry. The advantage of this extended LRSM over the generic one is that, this model accommodates low scale inverse seesaw mechanism for the origin of neutrino mass which also allows large light-heavy neutrino mixing. This mixing facilitates the interaction of left-handed gauge boson $\left(W_{L}\right)$ with heavy neutrinos and sizeable contributions to muon anomalous magnetic moment arises from this channel. The new $Z_{\mu \tau}$ boson associated with $U(1)_{L_{\mu}-L_{\tau}}$ symmetry also helps in ameliorating $\Delta a_{\mu}$ when it gets mass around $150 \mathrm{MeV}$. Moreover our predictions on the mass of $Z_{\mu \tau}$ and its coupling $g_{\mu \tau}$ lie well below the constraint given by ref [17]. For a detailed discussion on neutrino mass, mixing, new contributions to muon (g-2) anomaly in the model and its numerical calculation one may refer [18].

The model is governed by the gauge group,

$$
\mathbb{G}_{\mathbb{L}}^{\mu \tau} \equiv S U(2)_{L} \times S U(2)_{R} \times U(1)_{B-L} \times S U(3)_{C} \times U(1)_{L_{\mu}-L_{\tau}}
$$

In the model, new contributions to muon $(g-2)$ anomaly arise from the interactions of; (i) singly charged gauge bosons with heavy neutral fermions, (ii) neutral vector boson with singly charged fermions, (iii) singly charged scalars with neutral fermion, (iv) neutral scalars with muons, (v) extra light new gauge boson $Z_{\mu \tau}$ with muons.

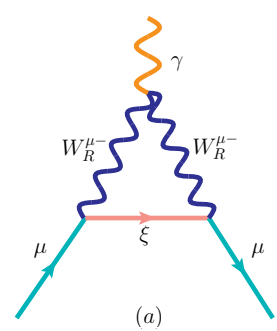

$(a)$

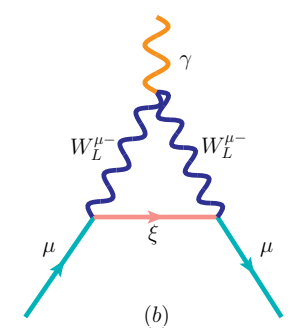

(b)

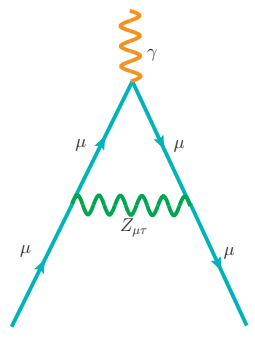

Figure 1: Feynman diagrams for the interaction of singly charged vector bosons $W_{L}, W_{R}$ with heavy neutrino and new neutral gauge boson $Z_{\mu \tau}$ with muon.

In the plots we have shown how the contribution of each particle to $\Delta a_{\mu}$ varies with the mass of that particle for different choices of couplings. Clockwise from top left, the first plot shows the variation of $\Delta a_{\mu}$ coming from purely left-handed currents via $W_{L}$ mediation with respect to the light-heavy mixing parameter $V^{v \xi}$. We have varied the mixing from $10^{-2}$ to 1 . The magenta line represents the dependence of $\Delta a_{\mu}$ on light-heavy mixing and we find that the mixing should be order 

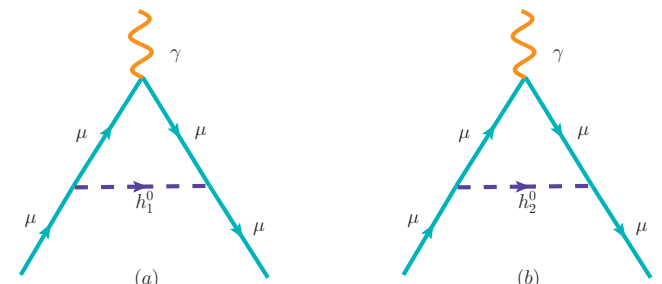

Figure 2: Feynman diagrams for the interaction of neutral CP-even scalars $h_{1}^{0}, h_{2}^{0}$ with muons.
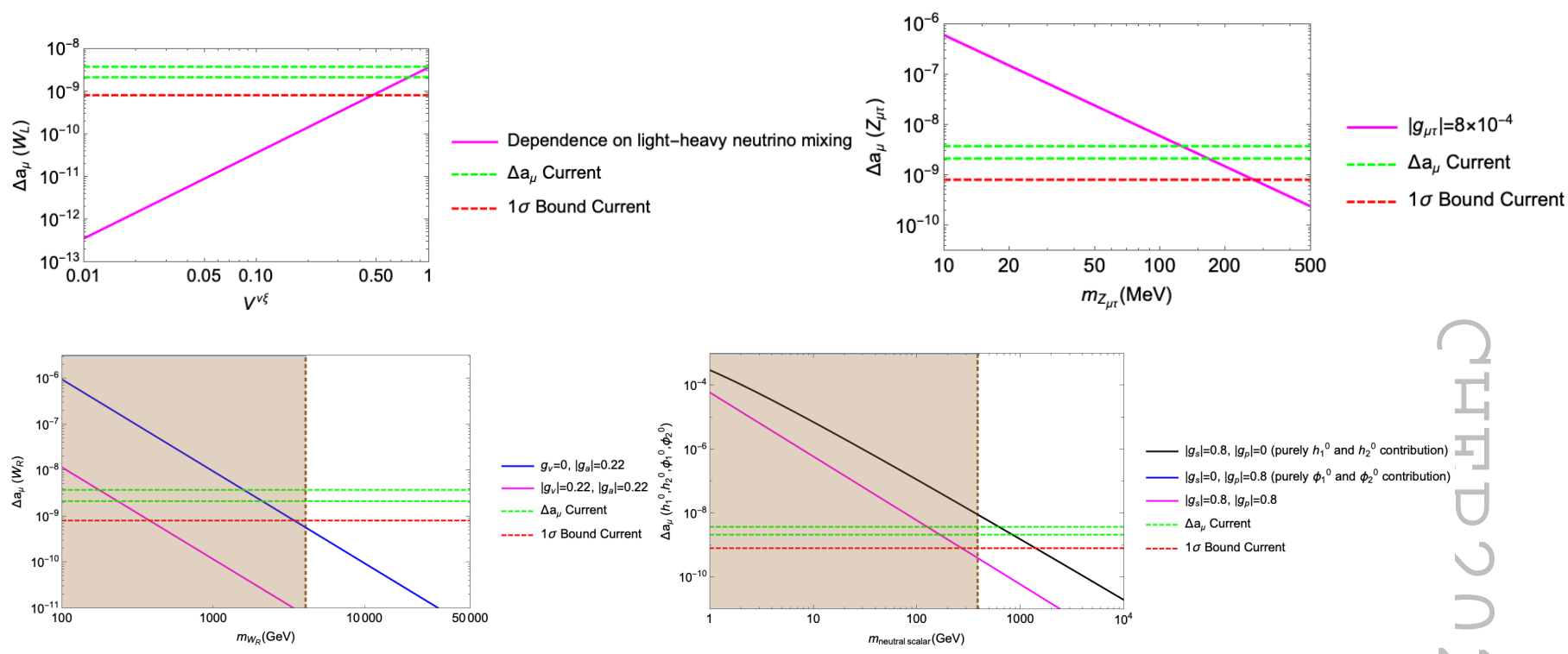

of $(0.3-1)$ in order to satisfy the current bound on $\Delta a_{\mu}$. The second plot shows the contribution of $Z_{\mu \tau}$ to $\Delta a_{\mu} v s$ its mass. The magenta line shows that $Z_{\mu \tau}$ with mass $150 \mathrm{MeV}$ and coupling $g_{\mu \tau}=8 \times 10^{-4}$ can address the anomaly. The third plot shows the combined contributions of neutral scalars to $\Delta a_{\mu} v s$ their mass. The shaded region shows the excluded mass range for neutral scalars $(<500 \mathrm{GeV})$. Thus neutral scalars with mass range $500 \mathrm{GeV}$ to $2 \mathrm{TeV}$ can explain the anomaly. The last plot shows the contribution of $W_{R}$ to $\Delta a_{\mu} v s$ its mass. We have considered various couplings to see which is sensitive to the current bound on $\Delta a_{\mu}$. It shows, with purely axial vector-like coupling (blue line) $W_{R}$ can address the anomaly, but its mass lies around $2 \mathrm{TeV}$ which doesn't satisfy the constraints on its mass. The shaded region indicates the excluded mass range of $W_{R}$.

From this study we found that $W_{L}$ with $O(1)$ light-heavy neutrino mixing, the new neutral vector boson $Z_{\mu \tau}$ and low-mass CP-even scalars are good candidates for explaining the anomaly. Overall we found that inverse seesaw mechanism influences the results on muon anomaly to a large extent while explaining neutrino mass and mixing in the model.

\section{References}

[1] M. Tanabashi et al. [Particle Data Group], Phys. Rev. D 98, no.3, 030001 (2018)

[2] T. Blum, A. Denig, I. Logashenko, E. de Rafael, B. L. Roberts, T. Teubner and G. Venanzoni, [arXiv:1311.2198 [hep-ph]]. 
[3] G. W. Bennett et al. [Muon g-2], Phys. Rev. D 73, 072003 (2006)

[4] P. S. B. Dev, W. Rodejohann, X. J. Xu and Y. Zhang, JHEP 05, 053 (2020)

[5] F. Jegerlehner and A. Nyffeler, Phys. Rept. 477, 1-110 (2009)

[6] M. Lindner, M. Platscher and F. S. Queiroz, Phys. Rept. 731, 1-82 (2018)

[7] H. Davoudiasl, H. S. Lee and W. J. Marciano, Phys. Rev. D 89, no.9, 095006 (2014)

[8] C. Kelso, P. R. D. Pinheiro, F. S. Queiroz and W. Shepherd, Eur. Phys. J. C 74, 2808 (2014)

[9] N. A. Ky, H. N. Long and D. Van Soa, Phys. Lett. B 486, 140-146 (2000)

[10] C. A. de S.Pires and P. S. Rodrigues da Silva, Phys. Rev. D 64, 117701 (2001)

[11] P. Agrawal, Z. Chacko and C. B. Verhaaren, JHEP 08, 147 (2014)

[12] W. Altmannshofer, C. Y. Chen, P. S. Bhupal Dev and A. Soni, Phys. Lett. B 762, 389-398 (2016)

[13] S. Jana, V. P. K. and S. Saad, Phys. Rev. D 101, no.11, 115037 (2020)

[14] M. Endo and W. Yin, JHEP 08, 122 (2019)

[15] A. Dev, [arXiv:1710.02878 [hep-ph]].

[16] W. Altmannshofer, S. Gori, M. Pospelov and I. Yavin, Phys. Rev. Lett. 113, 091801 (2014)

[17] A. DiFranzo and D. Hooper, Phys. Rev. D 92, no.9, 095007 (2015)

[18] C. Majumdar, S. Patra, P. Pritimita, S. Senapati and U. A. Yajnik, JHEP 09, 010 (2020) 